when they leave the existing expected ratio of second strike strengths unaltered, we can find an expression for this ratio if we can write the numbers of second strike aircraft as $P_{A}^{0}$ and $P_{B}^{0}$ where

$$
\begin{aligned}
& P_{A}^{0}=P_{A}\left(1-c_{A}\right)^{f_{B} P_{B} p_{A} / P_{A}} \\
& P_{B}^{0}=P_{B}\left(1-c_{B}\right)^{f_{A} P_{A} p_{B} / P_{B}}
\end{aligned}
$$

and $P_{A}, P_{B}$ are the existing numbers of aircraft; $c_{A}, c_{B}$ the probabilities that on average $p_{A}$ and $p_{B}$ aircraft will be destroyed before take off by one aircraft of the forces of $B$ and $A$ respectively; and $f_{A}, f_{B}$ are the fractions of the airforces of $A$ and $B$ devoted to a first strike disarming attack.

Then dividing equation (15) by equation (16) and differentiating we obtain after some manipulation

$$
\mathrm{d} P_{A} / \mathrm{d} P_{B}=P_{A} / P_{B}
$$

which becomes, after equating the small peacetime reductions in aircraft numbers $\Delta P_{A}$ and $\Delta P_{B}$ to the differentials,

$$
\Delta P_{A} / P_{A}=\Delta P_{B} / P_{B}
$$

from which and from equations (15) and (16) it also follows that

$$
\Delta P_{A}^{0} / P_{A}^{0}=\frac{\Delta P_{B}^{0}}{P_{B}^{0}}=\Delta P_{A} / P_{A}
$$

Thus not only are reductions in numbers of aircraft balanced in the sense of this section when they are carried out in equal percentages according to equation (18) but also, provided they are accompanied by numerically equal percentage reductions by both sides in troops and tanks, the new, smaller second strike tactical aircraft forces will automatically be in the same ratio to the new number of military targets (troops, tanks and aircraft) possessed by the other side as are the existing second strike forces to the existing number of military targets. (This will not be precisely true since there are second strike targets for tactical aircraft, such as bridges, fortifications and certain townships, the number of which will scarcely, or only indirectly, be affected by reductions in military equipment and personnel.) This important result clarifies and supports the original assumption that tactical aircraft reductions which preserve in being the existing ratio of second strike forces could be taken as balanced by the parties involved.

As a prescription for the way in which "balanced" force reductions in Europe should be carried out this paper does not pretend to completeness. In particular it does not deal with the question of how large such reductions should be, only their inter-relationships, although the technique of analysis is usually based on a presumption of small, up to $10 \%$, reductions.

This article sets out to put the case for equal or near equal percentage cuts as being most likely to preserve the existing balance of conventional military strength, over the wide spectrum of meanings given to that phrase. And the plausibility of this proposition gains considerably from the consistency with which it emerges from the three approaches to the problem.

Work on an extended version of this article is in progress. I thank R. P. Barston and R. N. Fournier for valuable conversations.

Department of Politics, University of Lancaster, Bailrigg, Lancaster

Received July 26; revised August 28, 1971.

${ }^{1}$ Richardson, L. F., Arms and Insecurity (edit. by Rashevsky, N., and Trucco, E.), 370 (Stevens, London, 1960).

${ }^{2}$ Saaty, T. L., Mathematical Models of Arms Control and Disarmament, 48, 49, 51 (Wiley, New York and London, 1968)

${ }^{3}$ Ogorkiewicz, R. M., Design and Development of Fighting Vehicles, 140 (Macdonald, London, 1968).

4 The Military Balance 1970-71, 94 (Institute for Strategic Studies, London, 1970).

${ }^{5}$ See Blackett, P. M. S., Studies of War, 198 (Oliver and Boyd, London, 1962).

\section{Supernatural Beliefs of Graduate Students}

IN their recent report entitled "Supernatural Beliefs Among Graduate Students at the University of Pennsylvania"1, Salter and Routledge examined the hypothesis that further education leads to a decline in supernatural beliefs, an idea already tested in a population of undergraduate males ${ }^{2}$. Their sample, however, was strongly biased in favour of male students with one year of graduate study and limited to married students living in a university campus residence hall, and therefore was unrepresentative of the total graduate student population. Of the 9,000 graduate students, approximately $50 \%$ are married and, of these, only $10 \%$ live in university campus housing.

We doubt the precision of the method used to measure the extent of an individual's belief in the supernatural. Each subject was rated on a scale from 0 to 20 according to his assessment of strength of his belief in certain phenomena. It is not clear if he was rated by himself or by the interviewer. Zero indicated total unqualified disbelief, 10 indicated neither belief nor disbelief, and twenty indicated total unqualified belief ${ }^{2}$. This rating could not be used to meaningfully compare different individuals, nor does the same score in different categories suggest an equivalent level of belief. For example, the authors would propose that a score of 20 for belief in the power of prayer has an equivalent relationship to the Gestalt of supernatural belief as does a score of $\mathbf{2 0}$ for belief in unidentified objects (UFOs). Similarly, a score of 10 on UFOs might suggest either an open mind on the subject of extra-terrestrial life, or an intermediate level of belief in something supernatural.

The most egregious errors, however, were in the analysis. Salter and Routledge, as well as the previous workers they cited, assumed that their scores represented interval rather than ordinal measures. That is, they assumed that a score of 10 on a particular item represented twice the belief as did a score of 5. There is no basis whatever for this assumption, as their 20-point scale was an arbitrary one. They calculated, however, the means of the scores for each of their eight items (astrology, ESP and so on), an operation which is invalid for ordinal data.

They then tested the hypothesis that amount or field of education decreases supernatural belief by measuring concordance of year or of field of study with the mean scores of the eight items. Their method of testing these hypotheses assumed, for example, that both belief in prayer and in UFOs were, in some sense, equivalent components of supernatural belief, and, therefore, that the scores on each should be concordant. Yet, as discussed, this is an unreasonable assumption. Their failure to find significant Kendall coefficients of concordance could have been as much due to true lack of concordance among the (mean) scores of the various items, as to the absence of trends in these items over years or fields of graduate study.

Finally, the authors made a number of contradictory assertions. For example, they write: ". . . natural and [sic] biological scientists had the highest mean belief scores and students in the arts . . . had the lowest. . . . These differences [were] insignificant . . ." Furthermore, descriptions of procedure were vague: "Our interviewers were instructed to explain the questions if problems arose", and the authors remark without additional comment: "Cell frequencies do not in every case add to the total because some data were improperly transcribed".

ROBERT M. FACTOR RICHARD MEYER

Department of Biology, University of Pennsylvania, Philadelphia, Pennsylvania 19104

Received August 17, 1971.

${ }^{1}$ Salter, C. A., and Routledge, L. M., Nature, 232, 278 (1971).

2 Pasachoff, J. M., Cohen, R. J., and Pasachoff, N. W., Nature, 227, 971 (1970). 